\title{
Electricity Safety Analysis and Improvement Based on User Classification
}

\author{
Hongxia zhu ${ }^{1 *}$, Qing Wang ${ }^{1}$, Congcong $\mathrm{li}^{1}$, Yan $\mathrm{Du}^{1}$, and Chao $\mathrm{Yu}^{2}$ \\ ${ }^{1}$ State Grid Shandong Electric Power Research Institute, Jinan, Shandong, 250000, China \\ ${ }^{2}$ ZhongShiYiTong Group, Jinan, Shandong, 250000, China
}

\begin{abstract}
Analyzing electricity consumption behavior by classifying user groups and knowing each group's characteristics are an important way to realize intelligent power consumption. This paper firstly classifies different types of users based on power consumption data and grasps the power consumption behavior of typical users. The main causes of accidents and alarms are then discussed based on the recorded events and power consumption curve. Finally, the corresponding solutions, which take the user's social attributes and electricity consumption behavior into consideration are put forward to avoid the potential accidents.
\end{abstract}

\section{Introduction}

In recent years, with the serious shortage of energy and environment deterioration, China has taken energy saving and emission reduction as the main objective in economic structure adjustment, and has raised it to the national level.

With the advancement of power system reform and energy Internet strategy, more and more power users purchase electricity and use related value-added services from internet, thus forming different kinds of data, including user attribute data, behavior data and online behavior data [1]. Accurately grasp the characteristics of electricity user's behavior through big data analysis could not only help government, industry and commerce departments to improve service level, but also improve the profitability and competitiveness of enterprises[2]. In integrated energy service, mastering energy consumption data and carrying out smart energy consumption plan for users can greatly improve the energy efficiency and economic benefits of the park [3].

Integrated energy service is one of the most important ways to push forward energy revolution and promote green and low carbon development of power industry. [4] analyzes the energy efficiency of each unit in the park based on measured data and also proposes an optimal power consumption strategy to make the park operate in the most efficient energy utilization way. [5] gives an overview of a variety of index to evaluate a park's energy efficiency. With the maturity of big data technology and the increase of energy demand, more and more enterprises put forward a variety of business models and innovative ideas for smart energy cloud services. It can be seen that energy use analysis and smart energy consumption plan play an important role in increasing energy efficiency in the park and promoting the power reform. [6]
This paper firstly analyzes the power consumption characteristics of different kinds of users based on collected data and discusses the causes of energy safety issues in the park. Based on the analysis results, the corresponding solutions are proposed to avoid accidents.

\section{Scenario Depiction}

The scenario studied in this paper has residential users, industrial users and commercial users. The commercial users can be roughly divided into three parts: retail, entertainment and office. Due to the different composition and operation of commercial systems, the power consumption will also be different [7]. Industry in the largest power consumption field at present, mainly including motor manufacturing, steel, chemical industry, flour manufacturing and other industrial power consumption. The power consumption data of all kinds of users are recoded by the terminal meter and uploaded to the smart energy cloud platform. This platform integrates big data technology, cloud computing, Internet of things, artificial intelligence and other technologies, and provides intelligent energy management services, including energy safety, economic energy utilization and refined energy management. The data listed in this paper are all from this cloud platform.

This platform can monitor voltage, current, reactive power in real time and detect energy safety hazard event. In addition, the cloud platform provides unified energy management and scheduling of information service, reactive power compensation service, intelligent metering service and electricity security warning service, etc. Among them, the detection items in security warning service includes voltage and current unbalance, voltage and current over limit, abnormal power factor, and

*Corresponding author's e-mail: 2008anewbegin@sina.com 
leakage, etc. the second chapter will analyze the causes of the accidents based on energy consumption curve.

\section{Energy Safety Analysis Based on User Classification}

\subsection{User Classification and Characteristics}

Table 1 shows the power consumption situation in 2019 of several industrial and commercial users in the park:

Table 1. Power consumption in 2019.

\begin{tabular}{cccc}
\hline users & industry & $\begin{array}{c}\text { Transformer } \\
\text { capacity }(\mathrm{kVA})\end{array}$ & $\begin{array}{c}\text { Annual energy } \\
\text { consumption }(\mathrm{kWh})\end{array}$ \\
\hline Large industrial user1 & battery & 1000 & 6993714 \\
Large industrial user2 & steel & 630 & 8535182 \\
Small industrial user3 & fertilizer & 250 & 370817 \\
Commercial user1 & office & 100 & 11860.2 \\
Commercial user2 & hotel & 200 & 172592.4 \\
Commercial user3 & office & 250 & 293688 \\
\hline
\end{tabular}

Among them, the load curves of large industrial user1 and small industrial user during a week (Jan. 1, 2020 to Jan. 08, 2020) are shown in figure 1 and figure 2 respectively (Jan. 1st ,4th, 5th are holidays and the rest are working days):

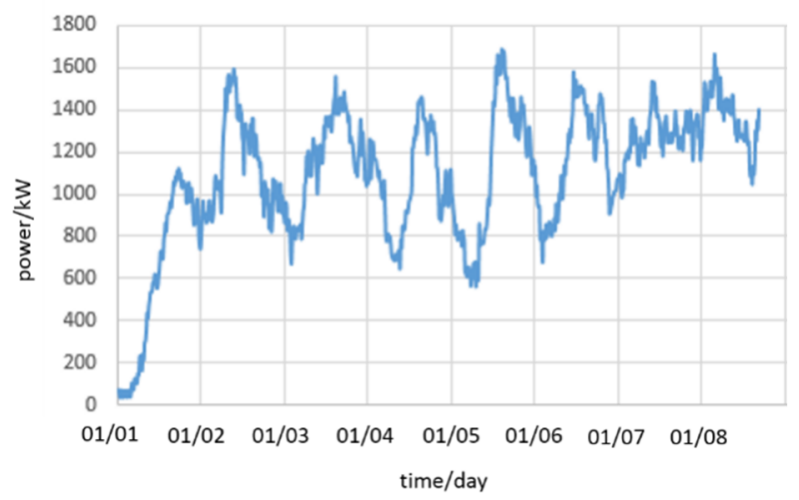

Figure 1. power consumption curve of large industrial user1.

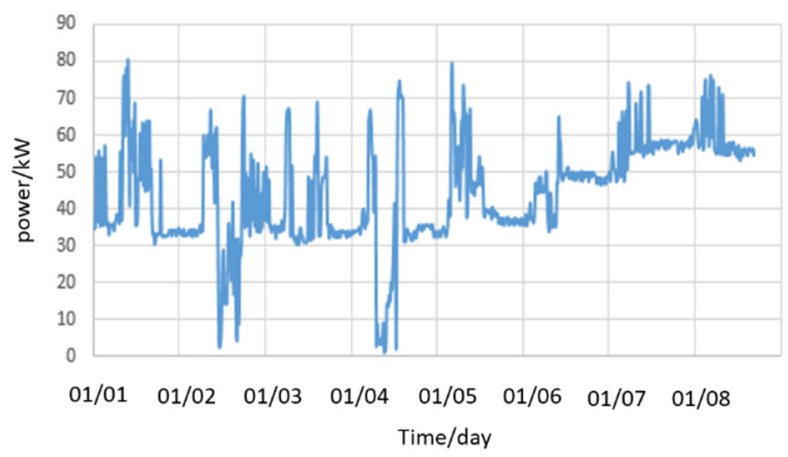

Figure 2. power consumption curve of small industrial user2.

Industrial power consumption has two characteristics: large power consumption and relatively stable power consumption [8]. Different industrial users have different power consumption characteristics due to the different industrial operation. For example, through data analysis, it can be found that the smelting industry in the park has a large, stable load and the load rate is high. However, the machinery manufacturing industry and food processing industry have a small load and the load rate is relatively low. The difference between these users' characteristics is mainly caused by the different production shifts.
The power consumption curves of commercial user 2(hotel) and a residential building on Sep. 1, 2019 are shown in figure 3 and in figure 4, respectively.

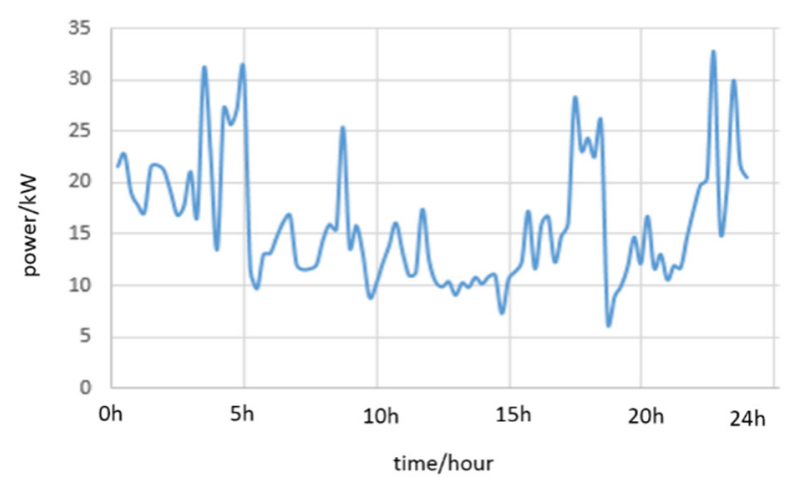

Figure 3. power consumption curve of a hotel.

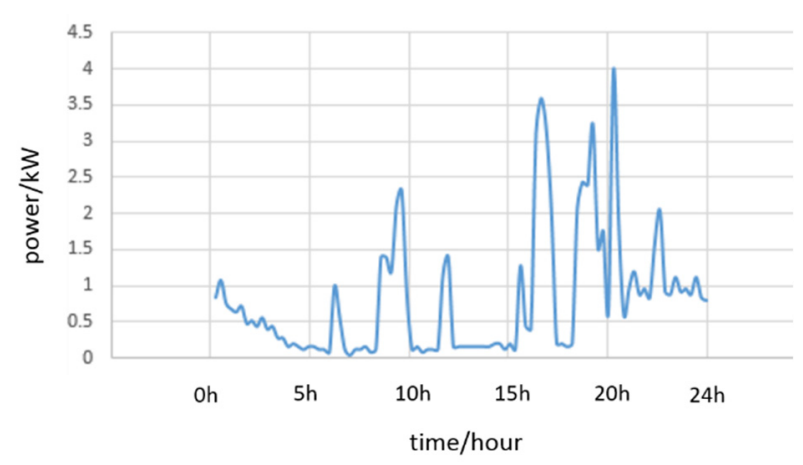

Figure 4. power consumption curve of a residential building.

Office load characteristic has a tight link with employee's work and rest schedule and has a strong regularity [9]. The power consumption at work is relatively high and stable, and will be significantly less after work. The load rate is even lower during holidays. This kind of load curve fluctuates obviously with seasons. The load rate is obviously higher when the outside temperature is too low or too high. However, this kind of load doesn't account for a high proportion of the total load in this park and the power consumption usually happens in daytime, which has little impact on the grid loading rate.

Residential electricity consumption is related to people's living habits [10], peak hours are at noon and in 
the evening, and the maximum demand generally occurs between 19:00 and 20:00.

\subsection{Energy Safety Events}

Up to now, most of the safety events recorded in the park are abnormal power factor, and a few of them are voltage out of limit, the imbalance of voltage and current and leakage. Table 2 and table 3 show the recorded abnormal power factor events of a large industrial user and a commercial user (hotel) respectively. By default, the platform will give a warning when the power factor is lower than 0.93 , and the calculation period is 24 hours.

Table 2. abnormal power factor events of a large industrial

\begin{tabular}{ccc}
\multicolumn{3}{c}{ user. } \\
\hline Terminal name & time & Power factor \\
\hline 1250kVA & $2020-01-23$ & 0.905 \\
630kVA & $2020-01-23$ & 0.552 \\
630kVA & $2020-01-24$ & 0.552 \\
1250kVA & $2020-01-25$ & 0.907 \\
630kVA & $2020-01-25$ & 0.496 \\
\hline
\end{tabular}

Table 3. abnormal power factor events of a commercial user.

\begin{tabular}{ccc}
\hline Terminal name & time & Power factor \\
\hline hotel & $2020-02-05$ & 0.9143 \\
hotel & $2020-02-06$ & 0.9117 \\
hotel & $2020-02-13$ & 0.8925 \\
hotel & $2020-02-14$ & 0.7982 \\
hotel & $2020-02-15$ & 0.8532 \\
\hline
\end{tabular}

When the power factor is low, the user will absorb reactive power from grid, which will reduce the output of the generator and change the grid node voltage, so as to increase the line current, line power loss and electricity bill. In addition, if the power factor of users, of which the capacity is above $100 \mathrm{~kW}$, is smaller than the standard $(0.9)$, the power supply company will have a higher operation cost and power user need to pay power factor adjustment fee, which make both sides not profitable.

In addition to abnormal power factor, there are also some other recorded issues, like voltage out of limit, voltage and current unbalance and leakage events. Table 4 and 5 list the leakage event and voltage imbalance event of a commercial user.

Table 4. leakage event of a commercial user.

\begin{tabular}{ccc}
\hline Terminal name & time & $\begin{array}{c}\text { Zero sequence } \\
\text { current }\end{array}$ \\
\hline Motor1(air & $2020-01-1710: 43$ & 1.433 \\
condition 1) & $2020-01-1710: 41$ & 1.711 \\
Lighting 1 & $2020-01-2210: 43$ & 0.027 \\
Lighting 1 (hall) & $2020-01-2202: 02$ & 0.033 \\
Lighting 1 & $2020-02-2516: 50$ & 9.628 \\
Motor1 & $2020-02-2516: 50$ & 0.112 \\
Motor1(air & \\
condition 2) & & \\
\hline
\end{tabular}

Table 5. voltage and current events of a commercial user.

\begin{tabular}{ccc}
\hline Terminal name & time & event \\
\hline Lighting 1 & $2020-01-22$ & Voltage over the \\
(lighting) & $02: 02$ & upper limit
\end{tabular}

\begin{tabular}{ccc} 
Lighting 1 & $2020-01-22$ & $\begin{array}{c}\text { Unbalance of voltage } \\
\text { (lighting) }\end{array}$ \\
$02: 02$ & and current \\
Lighting 1 & $2020-01-22$ & $\begin{array}{c}\text { Phase A voltage over } \\
\text { the upper limit }\end{array}$ \\
(meeting room) & $02: 02$ & Phase A voltage over \\
Lighting 1 (tea & $2020-01-22$ & the upper limit \\
room) & $02: 02$ & Voltage over the \\
Lighting 1 & $2020-03-10$ & upper limit \\
(lighting) & $11: 30$ & Unbalance of voltage \\
Lighting 1 & $2020-03-10$ & and current \\
(lighting) & $11: 30$ & \\
\hline
\end{tabular}

The action current of household leakage switch is $30 \mathrm{~mA}$. It can be seen from table 4 that a large leakage current event occurred on Jan. 17th and Feb. 25th. The voltage and current imbalance and out of limit events recorded in table 5 occur in the equipment lighting 1 . Lighting 1 is the main bus meter, hall lighting and hardware is 3 phase load and the rest are single-phase load.

In table 5, the lighting 1 , hall lighting, conference room, exhibition hall and hardware have all been recorded with voltage safety issues at 2:02 on Jan. 22nd. Among them, the current imbalance of PD lighting and hall lighting reaches $200 \%$ and $240 \%$ respectively.

\subsection{Reason Analysis of Recorded Events}

\subsubsection{Power Factor Analysis}

Figure 5 shows the active power curve, reactive power curve and power factor curve of $630 \mathrm{kVA}$ transformer on Jan. 23rd of the large industrial user, which is listed in table 2 . The data sampling interval is 15 minutes.

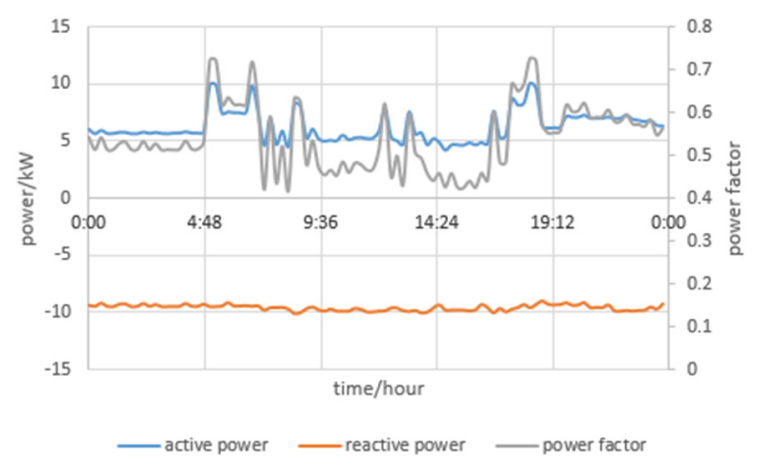

Figure 5. consumption curve on Jan. $23^{\text {rd }}$.

Figure 6 and figure 7 are the power curves of $800 \mathrm{kVA}$ transformer and $1250 \mathrm{kVA}$ transformer, which are all used by the same industrial user. 


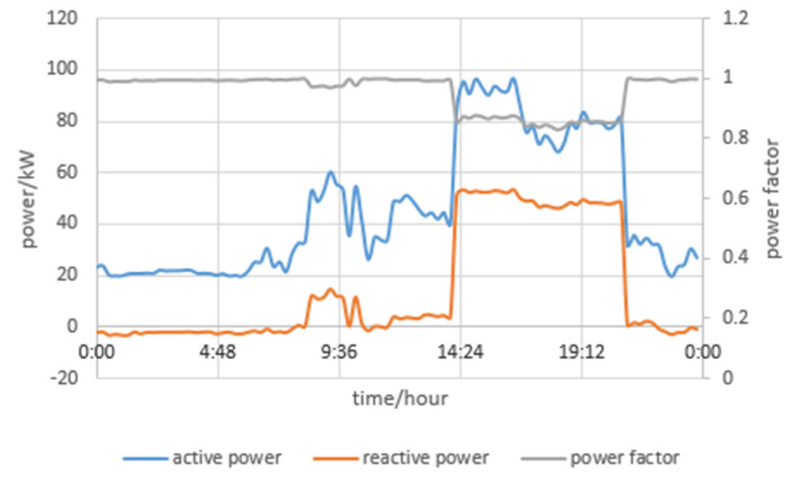

Figure 6. power curve under $800 \mathrm{kVA}$ transformer.

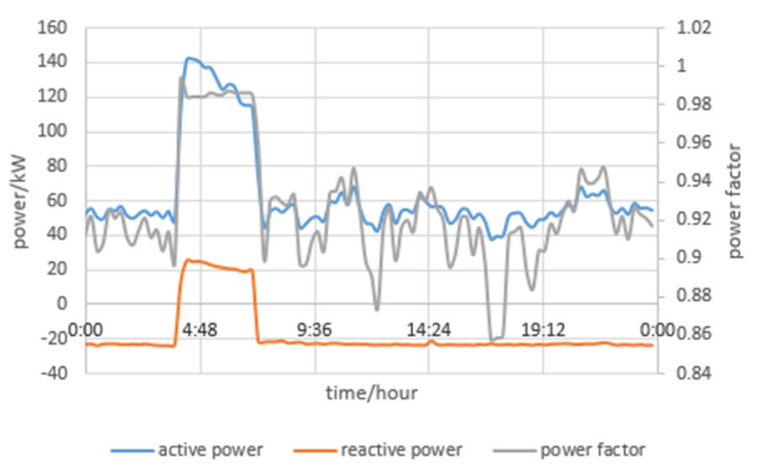

Figure 7. power curve under 1250kVA transformer.

Table 2 shows that there is no abnormal power factor issues under $800 \mathrm{kVA}$ transformer. The power factor under $630 \mathrm{kVA}$ transformer is lowest and the power factor is relatively low under $1250 \mathrm{kVA}$ transformer.

The electrical load in this enterprise is mainly inductive load. As the consumption changes all the time, once the inductive reactive power is lower than reactive compensation power, the recorded reactive power will be negative. In figure 5 , the reactive power is always negative and the active power is small, which lead to a low power factor. In figure 6 , the power consumption increases obviously, but the power factor is less than 0.9 in the peak load period, which is caused by the increase of inductive load and bad reactive power compensation strategy.

By comparing 3 curves, it can be found that large power consumption can improve power factor. The low loading rate and bad reactive power compensation strategy is the main reason for the low power factor of the industrial user.

\subsubsection{Analysis of Leakage, Voltage Imbalance and Over Limit}

The wiring plan of this commercial user adopts traditional TN-S three phase five-wire power supply system. During the normal operation, there is no current in the protection line and no unbalanced current in the zero line. Through data analysis, it can be found that three-phase imbalance and leakage events are usually happen at the same time. The unbalance of three-phase load results in the unbalanced current in the zero line [11].

The two voltage unbalance events occurred on Jan. 22nd and Mar. 10th, respectively. The maximum voltage imbalance is $0.3 \%$, resulting from PD lighting equipment and hall lighting. The unbalanced current of these two equipment are $200 \%$ and $140 \%$, respectively. PD lighting equipment is single-phase load and hall lighting is threephase device. Figure 8 is the power curve of hall lighting on Jan. 22nd, 2020

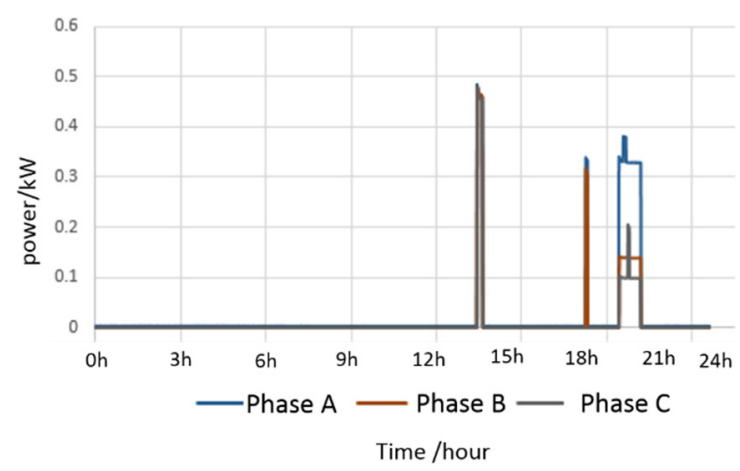

Figure 8. 3 phase power curve of hall lighting.

It can be seen from figure 8 that the consumption is very small and almost no load access to the grid. The voltage unbalance is not big and is not caused by singlephase grounding, which can be inferred from 3-phase voltage curve. The power supply system of this commercial user is composed of motive power and lighting, with a lot of single phase load. In the power supply system, single phase load makes three phase lines have different capacitance to the ground, so under normal circumstance, there is small inducted leakage current in $\mathrm{PE}$ in TN-S system. In addition, the connection of singlephase load will decrease the power consumption efficiency, which could aggravate three-phase load imbalance. Therefore, the unreasonable distribution of three-phase load and the access of a large number of single-phase load are the main reasons for the unbalanced voltage and current of this commercial user.

\section{Energy Using Strategy}

In order to improve power factor, the reactive power compensation on the user side should change in real time according to the load curve. The capacitor should be disconnected when there is no load or the load is small and put into operation as the apparent power increases. Secondly, try to use electrical equipment with a higher power factor to reduce the consumption of inductive reactive power. If the user can limit the power factor within the standard, the enterprise could save considerably electricity cost, and power supply company will have a lower line loss and a higher transformer capacity utilization rate, which could bring in a win-win situation.

As for the distribution of three-phase load, the staff is supposed to collect and record related power data, which could be used to predict consumption curve [12]. Installing a balance device could also realize a better three-phase balance. In addition, it is necessary to carry out three-phase load monitoring work, so as to detect three-phase unbalance event in time. The equipment with potential safety hazards should be adjusted properly. 


\section{Conclusion}

This paper analyzed power consumption characteristics of several users in a park in Shandong province based on the consumption data and discussed the reasons for energy safety events, including low power factor and unbalanced voltage and current. The advices to avoid these events are proposed in the last part.

To avoid unbalanced three phase voltage and achieve smart energy utilization, some more detailed information, like the system wiring topology of the user and each electrical device consumption curve are needed. Give user a more intelligent energy use strategy to avoid safety issues and reduce electricity cost will be our next research move.

\section{Acknowledgments}

This work was supported by science and technology project of State Grid Shandong Electric Power Company under Grant No. 520626190043.

\section{References}

1. Xu Changqing, Zhao Huadong, Song Xiaohui. (2016) Research on Identification and Analysis Method of Power User Group Based on Big Data. Journal of Zhengzhou University (Science Edition), 48 (03): 113-117.

2. Xin Miaomiao, Zhang delay, Xie Da. (2019) A review of research on consumer electricity consumption behavior based on big data of electricity. Electrical Automation, 41 (01): 1-4.

3. Wang Jingwen, Li Huaqiang, Li Xuxiang, Yi Lifeng, Bai Hongkun, Li Wenfeng. (2020) Utility Model of Comprehensive Energy Service and User Demand Evaluation. Chinese Journal of Electrical Engineering, 40 (02): 411-425.

4. Sun Yi, Jia Mengyang, Lu Jun, Sun Chenjun. (2017) An interactive evaluation model for residential electricity consumption in demand side management. Automation of Electric Power Systems, 41 (13): 62-69.

5. Wu Jinglian, Mu Yunfei, Jia Hongjie, Tian Yan, Niu Jide, Xu Jing. (2019) Energy efficiency index of multi-energy collaborative park based on energy value. Automation of Electric Power Systems, 43 (21): 54-63.

6. Hu Yan, Wen Yan, Liu Yuquan, CAI Ying, Yu Qi, Cheng Haozhong. (2019) Reliability Analysis of Integrated Energy System Based on User-side Energy Conversion Equipment. Journal of Electric Power Science and Technology, 34 (02): 11-19.

7. Zhang Xiaolong. (2017) Research on consumer electricity consumption behavior in big data environment. North China Electric Power University (Beijing).

8. Qi Bing, Zhai Tianyi, Li Bin, Yang Bin, Shi Kun, Li Dezhi. (2019) Research on Intelligent
Electricity Interactive Mode Based on Industrial Internet Cloud Platform. Electrical Measurement \& Instrumentation, 56 (19): 46-52.

9. Wang Qingze, Chen Xingying, Yan Qingguo, Xu Shiming, Liao Yingchen, Yu Kun. (2017) Doublelayer flexible optimization control strategy for office building air-conditioning load under timeof-use electricity price environment. Power System Protection and Control, 45 (21): 43-50.

10. Zhu Zhenyu. (2018) Residential electricity consumption behavior and evolutionary game under intelligent demand response. Southeast University.

11. Xu Yong. (2016) Research on Three-phase Load Imbalance Control System. Jiangsu University, 2016.

12. Zhang Yongjun, Liu Ziwen, Song Weiwei, Chen Guangjin, Tang Qingquan, Chen Boda. (2019) Networking Technology and Application of DC Power Distribution System. Automation of Electric Power Systems, 43 (23): 39-51. 